\title{
Development of a Community-Based Palliative Care Model for Advance Cancer Patients in Public Health Centers in Busan, Korea
}

\author{
Sook-Nam Kim, PhD, $\mathrm{RN}^{1}$ \\ Soon-Ock Choi, PhD, RN ${ }^{1}$ \\ Seong Hoon Shin, PhD, MD² \\ Ji-Sun Ryu, PhD, MSW ${ }^{3}$ \\ Jeong-Won Baik, PhD, MSW4
}

\section{${ }^{1}$ Department of Nursing, Catholic \\ University of Pusan, Busan, \\ ${ }^{2}$ Division of Hemato-Oncology, \\ Department of Internal Medicine, \\ Kosin University Gospel Hospital, Busan, \\ ${ }^{3}$ Department of Aging and Social Work, \\ Catholic University of Pusan, Busan, \\ ${ }^{4}$ Industry Academic Cooperation Foundation, \\ Catholic University of Pusan, Busan, Korea}

Correspondence: Seong Hoon Shin, PhD, MD

Division of Hemato-Oncology,

Department of Internal Medicine,

Kosin University Gospel Hospital,

262 Gamcheon-ro, Seo-gu, Busan 49267, Korea

Tel: 82-51-990-6107

Fax: 82-51-990-3005

E-mail: ssh1533@hanmail.net

Co-correspondence: Soon-Ock Choi, PhD, RN Department of Nursing, Catholic University

of Pusan, 57 Oryundae-ro, Geumjeong-gu,

Busan 46252, Korea

Tel: $82-51-510-0726$

Fax: 82-51-510-0747

E-mail: sochoi@cup.ac.kr

Received July 2, 2016

Accepted October 7, 2016

Published Online October 18, 2016

\begin{abstract}
Purpose
A feasible palliative care model for advance cancer patients is needed in Korea with its rapidly aging population and corresponding increase in cancer prevalence. This study describes the process involved in the development of a community-based palliative care (CBPC) model implemented originally in a Busan pilot project.
\end{abstract}

\section{Materials and Methods}

The model development included steps I and II of the pilot project, identification of the service types, a survey exploring the community demand for palliative care, construction of an operational infrastructure, and the establishment of a service delivery system. Public health centers (including Busan regional cancer centers, palliative care centers, and social welfare centers) served as the regional hubs in the development of a palliative care model.

\section{Results}

The palliative care project included the provision of palliative care, establishment of a support system for the operations, improvement of personnel capacity, development of an educational and promotional program, and the establishment of an assessment system to improve quality. The operational infrastructure included a service management team, provision teams, and a support team. The Busan Metropolitan City CBPC model was based on the principles of palliative care as well as the characteristics of public health centers that implemented the community health projects.

\section{Conclusion}

The potential use of the Busan CBPC model in Korea should be explored further through service evaluations.

Key words

Chronic disease, Neoplasm, Patients, Palliative care,

Pilot projects

\section{Introduction}

In Korea, $28.6 \%$ of all deaths are cancer-related, but only $13.8 \%$ of the population receives palliative care before death $[1,2]$. To improve palliative care, Korea has implemented policies aimed at increasing its use to $20 \%$, increasing the number of palliative care beds from 1,000 to 1,400 , and pro- viding a variety of palliative care services, including a palliative care consultation system and a home-based palliative care system by 2020 [1]. In addition, to build social consensus and systems for palliative care, a payment system was initiated in July 2015 to guarantee that the health insurance system covers the cost of inpatient palliative care for terminally cancer patients. The "Palliative Care and the Decision Regarding Life-Sustaining Treatment of End-of-life Patients 
Bill" was passed in 2016 and was set to be enacted in 2018 [3]. On the other hand, the Korean palliative care system is limited in usage and short in duration.

The scope of palliative care was recently expanded to include patients with end-stage heart disease, respiratory problems, kidney failure, liver diseases, and neurological disorders. With the increase in the elderly population, the inclusion of an increasing number of patients with non-cancer end-stage illnesses is expected to widen the scope of palliative care and the number of beneficiaries. Palliative care is not an end-stage care provided for patients for whom active treatment has been discontinued; rather, it involves physical symptom control and psychosocial and spiritual support. Therefore, it is an inclusive care approach that should begin at a life-threatening disease diagnosis [4]. Since the 2000s, the interest in palliative care has emerged from human rights and social justice perspectives, and the need for healthcare policy to ensure that every member of society benefits from the service has been emphasized [5]. The service should be easily accessible and universal to ensure that anyone can use it. In addition, a good strategy based on community involvement is required, along with an increase in community knowledge and public awareness of palliative care. The World Health Organization (WHO) suggests that palliative care strategies should combine palliative and primary care in communities that use public healthcare services [6].

This paper proposes that a community-based palliative care (CBPC) strategy be considered in end-of-life Korean care strategies because the use and accessibility of palliative care is particularly low there. In 2009, Busan, Republic of Korea, conducted a pilot CBPC project centered on public health centers (i.e., community-level primary healthcare facilities). Public health centers were selected as the focal points for allowing the use of various community resources investing in early approaches to end-of-life care and social support because they are public primary healthcare institutions at which community members first make contact and receive medical care. Palliative care provision must satisfy the fundamental concepts of healthcare delivery systems (i.e., accessibility, continuity, and standardized quality services). The Busan Metropolitan City CBPC pilot project, which was conducted between 2009 and 2014, established a basic framework for the structure, personnel, and service content required to provide $\mathrm{CBPC}$ based on other healthcare delivery systems [7]. In January 2015, Busan applied the system developed in the pilot project and began a CBPC project in the city's public health centers. To develop and standardize the CBPC service model, Busan created a CBPC center (CBPCC) that acted as a control tower and provided service standardization, directives, education, execution strategy development, and quality control.

This paper describes the processes involved in establishing the Korean CBPC model. The study aimed to develop a CBPC model based on the Busan CBPC pilot project, the demand for palliative care, and previous studies. The specific objectives were as follows: to determine the community demand and identify suitable types of CBPC services, to establish an operational infrastructure for CBPC task performance, and to establish a CBPC service delivery system.

\section{Development of CBPC Model}

The model was developed during steps I and II of the pilot project. The developmental process of the CBPC model included identification of the types of services required to meet the CBPC demand, and the establishment of an operational infrastructure and service delivery system. The study received financial support from Busan and the Korean Ministry of Health, Welfare, and Family Affairs Research and Development Fund. The study was approved by the Catholic University of Pusan Research Ethics Committee (CUPIRB2014-059).

\section{Geographical region and target population}

The project was conducted in Busan, the second largest city in Korea, which has a population of 3.41 million (2014) and an area of $764.43 \mathrm{~km}^{2}$. The city includes 16 districts and counties. In 2014, the Korean cancer mortality rate was 150.9 per 100,000 people per year, and that of Busan Metropolitan City was 175.2 per 100,000 people per year, the highest rate in Korea [8]. The four palliative care centers in Busan provide a total of 87 beds, which represents $49 \%$ of the total number of beds required based on the criterion that stipulates 25 beds per 500,000 people [9]. Korean palliative care centers currently provide only inpatient services, and palliative care staff members include physicians who have completed 60 hours of standardized training in palliative care, as well as palliative nurses, social workers, chaplains, and volunteers who have received 30 hours of training.

As the study aimed to develop a CBPC model centered on public health centers, a system for service provision was established by selecting home-based patients receiving endof-life care who were registered at the public health centers as the target population. Public health centers were selected as the regional hubs for CBPC model development because they have sufficient medical personnel, community resources, and networks, and the local residents can access them readily. The scope of the project and characteristics of the target population were as follows:

- The study included home-based patients receiving end- 
of-life care, who were registered at Busan public health centers between January and December 2015, their families, and their service providers.

- Sixteen Busan public health centers served as regional hubs for CBPC model development.

- Regional cancer centers, palliative care centers, and social welfare centers in Busan were involved in model development.

- The independence of each organization was respected. On the other hand, the CBPCC that housed the model development team was responsible for research/development decision making and regulation.

\section{Model development team}

The initial model development team consisted of two nursing professors with expertise in palliative care. These experts proposed a palliative care project centered on public health centers and conducted a pilot project from 2009 to 2014. In 2015, when the project was expanded to include all of Busan Metropolitan City, a full model development team, consisting of six professors in nursing, social work, business administration, and medicine, was formed.

Busan consigned the CBPC project to the model development team, which managed the planning and execution unit within the service management team. In particular, the model development team set the vision, goals, and strategies of the project and made decisions regarding the crucial aspects of model development involving infrastructure for execution, service standards, and outcome measures.

\section{Developmental steps}

\section{1) Pilot project step I (2009-2014)}

In 2008, a research team specializing in palliative care submitted a CBPC project proposal to the Health Improvement Department of Busan and persuaded the city's health administrators to support it. In 2009, the model development team was entrusted with the project and formed a research support committee. A pilot project center began in 2009 and included one public health center; however, the number of participating public health centers had increased to six by the 2014 project completion date. During the pilot project, Busan supplied administrative and financial support. The regional cancer centers provided technical support, and public health centers played the role of service providers. The model development team supervised, planned, and regulated the project.

During the study period, the model development team assembled an early framework for an operating model based on palliative care recipients, service level, labor standards for palliative care teams, personnel education, and consortium establishment [10]. Busan accepted the model development team's request for continuous adaptation, development, financial support, and evaluation based on the access of home-based cancer patients to palliative care and the effective use of healthcare resources. Furthermore, Busan recognized that the CBPC project increased the quality of life for home-based cancer patients and their families and was valuable as an end-of-life care system that was easily accessible to a highly vulnerable low-income population. Accordingly, in 2015, the city decided to expand the project to include public health centers across Busan.

\section{2) Pilot project step II (2015)}

Since January 2015, the CBPC project included 16 public health centers in Busan Metropolitan City as regional hubs and applied the preliminary operating model developed in Step Ito establish a CBPC model. The model development team requested a grant from the Ministry of Health, Welfare, and Family Affairs' Health Technology Research and Development Project for the development of a CBPC service system, for which it received 2 years of research funding for 2015 and 2016. Busan established the CBPCC to effectively conduct the CBPC project and entrusted the model development team with the responsibility of supervising, planning, and evaluating the project. The research support committee that operated during the pilot project was reorganized to include Busan regional cancer centers, the Social Service Network, legal experts, religious leaders, and journalists.

\section{Results of CBPC Model Development}

\section{Community demand for palliative care}

During step I, palliative care services were designed based on the demands of experts and home-based patients receiving end-of-life care in the community in which the pilot project had been conducted. In step II, 802 home-based cancer patients registered at public health centers in Busan and 651 center staff members to assess the palliative care needs were surveyed (Table 1).

Most home-based cancer patients $(68.8 \%)$ and public health center personnel $(96.6 \%)$ responded that they desired to receive palliative care services. The most preferred type of visiting care personnel was nurses $(85.5 \%$ of the home-based patients, and $83.4 \%$ of the community health center personnel), and the preferred types of home-based palliative care services were psychological/emotional support $(72.1 \%$ of 
Table 1. Demand for palliative care from public health center personnel and home-based cancer patients registered at public health centers ${ }^{\text {a) }}$

\begin{tabular}{|c|c|c|c|}
\hline Area & Item & $\begin{array}{l}\text { Home-based } \\
\text { cancer patients } \\
(\%)(\mathrm{n}=802)\end{array}$ & $\begin{array}{l}\text { Public health } \\
\text { center personnel } \\
(\%)(\mathrm{n}=651)\end{array}$ \\
\hline \multirow[t]{2}{*}{ Intention to use palliative care } & Use & 68.8 & 96.6 \\
\hline & Not use & 31.2 & 3.4 \\
\hline \multirow[t]{4}{*}{ Preferred home-based palliative care service provider } & Nurse & 85.5 & 83.4 \\
\hline & Volunteer & 17.9 & 36.7 \\
\hline & Social worker & 15.0 & 34.6 \\
\hline & Doctor & 14.5 & 24.7 \\
\hline \multirow[t]{6}{*}{ Preferred home-based palliative care service } & Psycho-emotional support & 72.1 & 82.6 \\
\hline & Medical support & 69 & 64.4 \\
\hline & Educational information & 60.6 & 57.4 \\
\hline & Socioeconomic support & 48.2 & 49.9 \\
\hline & Spiritual support & 6.9 & 41.9 \\
\hline & Family support & 4.7 & 50.2 \\
\hline \multirow[t]{4}{*}{ Preferred place of death } & Home & 49.4 & 63.7 \\
\hline & Hospital & 28.6 & 9.2 \\
\hline & Palliative care facility & 12.3 & 18.6 \\
\hline & Nursing care facility & 7.1 & 4.8 \\
\hline
\end{tabular}

${ }^{a)}$ Multiple response.

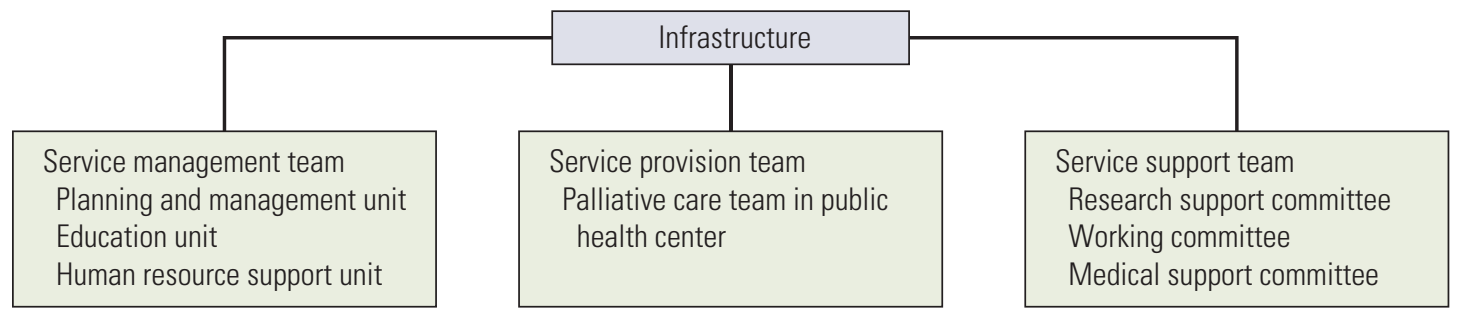

Fig. 1. Community-based palliative care service infrastructure.

patients and $82.6 \%$ of staff) and medical support (69\% of patients and $64.4 \%$ of staff). Home-based cancer patients preferred their homes $(49.4 \%)$ and hospitals $(28.6 \%)$, while public health center personnel preferred their homes $(63.7 \%)$ and palliative care facilities $(18.6 \%)$ as the places of death.

\section{Derived objectives and content}

The following goals and business plans for the CBPC project were established based on the pilot project and survey results regarding the service demands from the main project stakeholders.

The project's goals were to provide end-of-life, home- based hospices, and palliative care for patients and their families with public health centers as the community hubs, and to improve the quality of life for both patients and their families. The business plan was as follows.

(1) Provide CBPC

(2) Establish a support system for CBPC operation

(3) Train personnel involved in CBPC

(4) Develop educational and promotional programs to raise the CBPC awareness

(5) Create an assessment system to improve the CBPC quality 


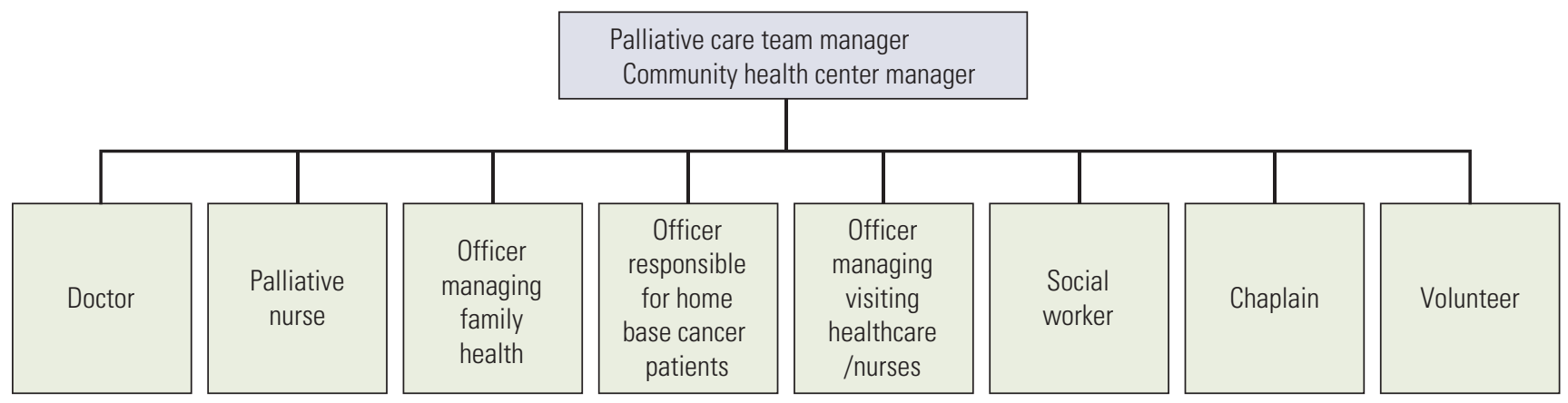

Fig. 2. Service provision team structure: public health center in Busan.

\section{Infrastructure organization}

The following groups were established to manage the CBPC project (Fig. 1).

\section{1) Service management team}

The service management team was a part of the CBPCC and included planning and management, education, and human resource support units.

The planning and management unit established the project's vision, goals, and strategies and made decisions regarding the infrastructure for execution, service content, and outcomes. The unit included palliative care coordinators, social workers, and six professors specializing in palliative care nursing, social work, and business administration. They held weekly planning and management meetings and made decisions regarding service direction.

The educational unit was responsible for providing the education and information required to allow the CBPC teams at 16 public health centers to perform their tasks. The unit included four professors specializing in palliative care, nursing practitioners in palliative care, social workers, and other expert consultants.

The human resource support unit was responsible for supporting the personnel required to provide CBPC. One of the responsibilities of the service management team was to support palliative nurses, social workers, and volunteers to ensure that public health centers could provide palliative care. The educational unit trained palliative nurses before dispatching them to public health centers, along with social workers and volunteers, when public health centers required staff or support. Because Korean public health centers implement several community healthcare projects and employ insufficient numbers of palliative care professionals, a system was established through which trained nurses and social workers were dispatched to maintain the palliative care qual- ity. Palliative nurses all had more than 2 years' hospital work experience and had either completed 60 hours of standardized training in palliative care or had obtained palliative care nursing certification. Social workers who had completed standard training in palliative care were also hired and were dispatched to public health centers where needed.

CBPCC staff that provides first-hand services consisted of a total of 13 members: nine palliative nurses, two palliative social workers, and two administrative workers.

\section{2) Service provision teams}

Palliative care teams were formed to provide comprehensive palliative care in public health centers. These teams consisted of a public health center manager, a physician, a palliative nurse, a social worker, a chaplain, and volunteers (Fig. 2) [10]. Public health center managers had the overall administrative responsibility. The physicians provided medical services, and the officer responsible for family health performed the administrative tasks. The staff working on the project for home-based cancer patients planned and executed the budget, handled the business aspects of palliative care provision in public health centers, and managed the volunteers. The manager of the healthcare home-visit team and home-visiting nurses identified and referred home-based cancer patients requiring palliative care to the palliative care team, and a chaplain provided spiritual support. Palliative nurses and social workers were responsible for case management. They also held weekly case management meetings with the service management team to prioritize the tasks and make decisions regarding resource distribution from a problem-solving perspective. The service provision team members were also responsible for other healthcare tasks in public health centers. CBPC project team meetings were held quarterly to allow the nurses to report the status of palliative care recipients and discuss the issues requiring resolution.

The CBPC project was based on the operating principles 


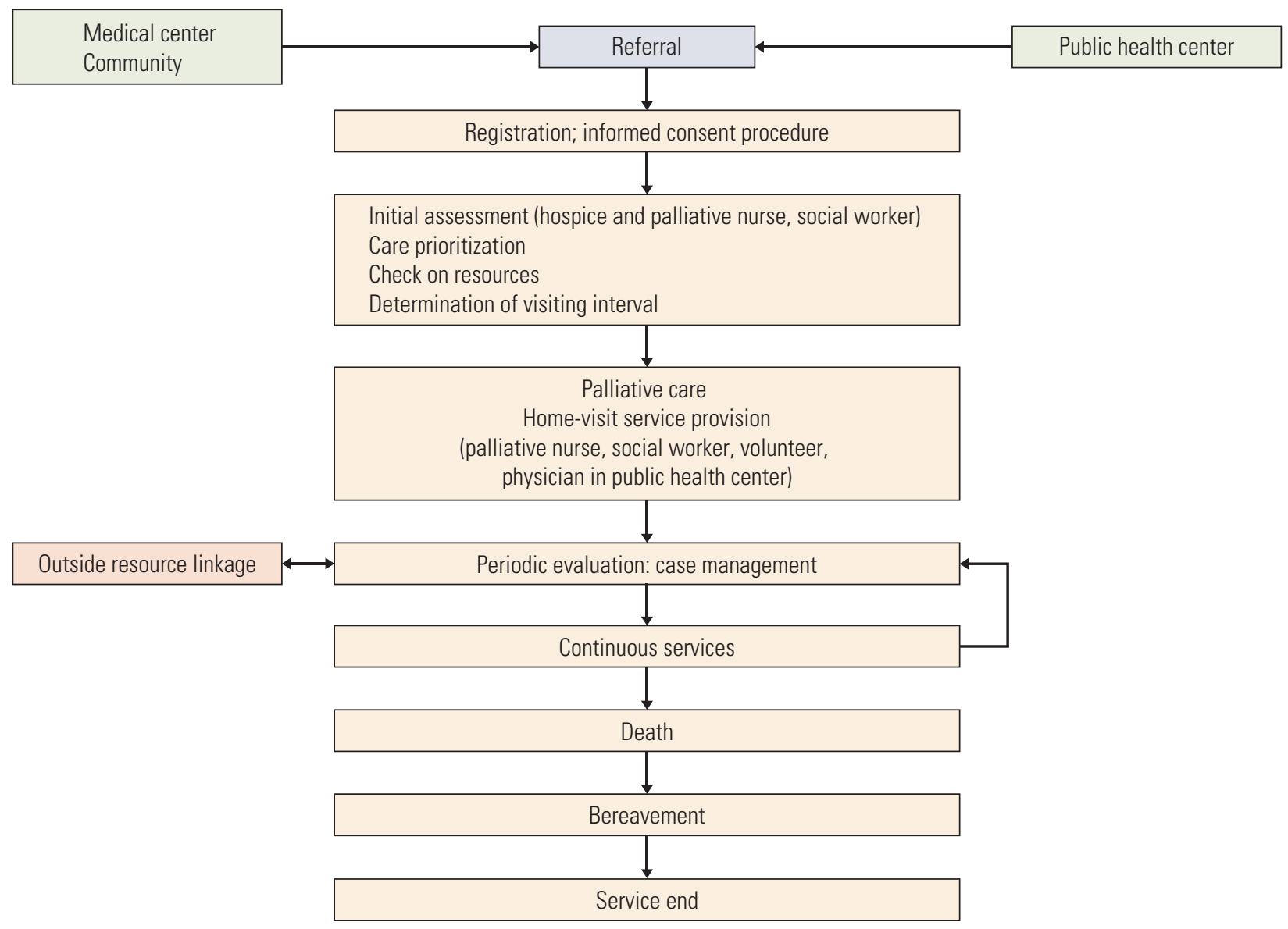

Fig. 3. Case management pathway.

for the effective utilization of community personnel and material resources with minimal investment from public health centers. Accordingly, the palliative care teams were established by adjusting the task assignments in public health centers, rather than hiring additional staff. On the other hand, palliative nurses and social workers who provided the care recipients with direct services were a part of the CBPCC and performed the tasks as service provision team members while receiving education and support in recipient management from the center. One palliative nurse was assigned to two public health centers and provided services by visiting the registered palliative care recipients at home. Two social workers were involved in managing cases to which patients at one of the 16 public health centers had been referred because of the socioeconomic need and the need for support from a palliative nurse (Fig. 2) [10].

Out of the 490 patients treated at the 16 health centers from January to December 2015, 485 (99\%) were terminally ill cancer patients and five (1\%) were terminally ill non-cancer patients.

\section{3) Service support team}

The service support team was responsible for the administrative, financial, and technical consultations. This team consisted of the research support, working, and medical support committees.

- The research support committee included Busan regional cancer center personnel, the Social Service Network, legal experts, religious leaders, and journalists. The committee discussed the project-related issues and mainly provided financial and administrative support. Committee meetings were held at least biannually.

- The working committee regulated and discussed everyday project execution and consisted of officers managing everyday CBPC project implementation at public health centers, palliative nurses, and service management teams in palliative care centers. The committee members held meetings at least biannually where needed.

- The medical support committee served as a support system providing medical service consultations at public 


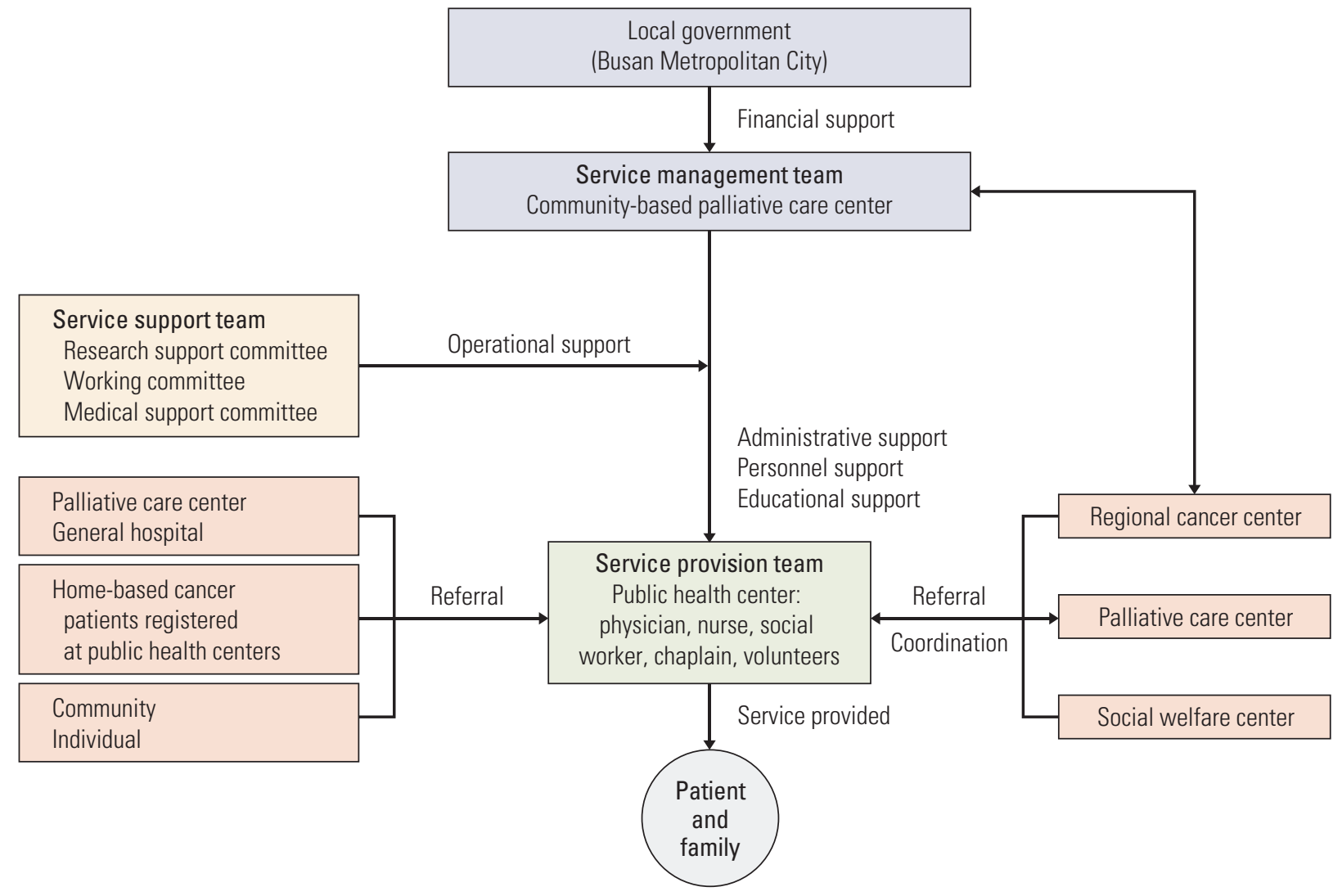

Fig. 4. Community-based palliative care service delivery system.

health centers to control the patients' symptoms and pain. The committee included three physicians who specialized in palliative care and were responsible for providing education and consultations regarding the management of end-of-life symptoms for public health center physicians.

\section{Case management and referral system}

\section{1) Case management pathway}

The specific case management pathways for palliative care recipients referred to service provision teams were as follows (Fig. 3).

The referred patients were registered as case management recipients by a palliative nurse after they gave informed consent. After registration, a palliative nurse and a social worker initially assessed patients' service demands and provided services according to prioritized care plans. The visiting intervals were determined according to the service demand, and a hospice volunteer was dispatched once or twice per week to provide physical, psychological, and spiritual help.
If community medical support was required, medical support from a physician and home visits were arranged by the service provision teams.

Palliative care recipients showing symptoms necessitating hospitalization while receiving palliative care were referred to a consortium palliative care center or hospital and treated as inpatients. Case management pathways were created to ensure that the patients would be referred back to the CBPC service provision teams if their symptoms improved and they were discharged. A bereavement service was provided when the care recipients died, and family members who were not at high risk of bereavement were removed from the system after 13 months.

Patients and families were provided with a phone number providing access to a 24-hour, on-call service for use when emergencies occurred or they required counseling outside of normal business hours. Palliative nurses were responsible for this service.

\section{2) Referral system}

The primary referral path for CBPC service recipients was 
accessed via home-visit teams or nurses working on a project for home-based cancer patients who referred patients registered in their systems to palliative nurses. In addition, patients and their families could contact the CBPC service office directly, and the social welfare center could refer patients who had been discharged from palliative care centers.

Patients referred to palliative nurses received care after providing informed consent and completing registration. A consortium system was established to ensure that the service provision teams referred patients requiring inpatient treatment to palliative care centers or the palliative medical ward of a regional cancer center in the consortium system. If professional services from a social welfare center were required, help was provided via a social economic support referral system. The referral system of a CBPC project was designed to ensure that the regional cancer and palliative care centers maintained close coordinating relationships with the service provision teams, and when the palliative care recipients required medical support, they were admitted to a consortium hospital and referred back to service provision teams upon discharge to continue receiving care (Fig. 4).

\section{CBPC service delivery system}

During the implementation of a pilot project between 2009 and 2015, the model development team established a service delivery system to provide services for patients requiring palliative care from public health centers (i.e., primary care organizations as regional hubs) (Fig. 4).

The service management team responsible for the project provided the personnel and technological and administrative support required by the service provision teams to manage the project. Public health centers established service provision teams and implemented the project with operational support from the service management team. The primary beneficiaries of the CBPC project included patients who required palliative care and were referred by a project team for home-based cancer patients or a healthcare home-visit team public health center. In addition, patients who were referred from regional palliative care centers, hospitals, or social welfare centers were registered and received comprehensive services from the service provision teams and volunteers following the initial assessment. Palliative nurses who worked with the public health centers to visit and provide direct care to patients discussed with the service management team in weekly case management meetings the type and level of care each patient required.

The service management team developed practical guidelines for the service provision teams at public health centers to provide standardized services and develop strategic plans for education, research, and promotion of the overall project.
The service provision teams identified patients, provided care, and offered direct services by coordinating the required community resources. Finally, the service support team aided the service provision teams regarding general issues concerning palliative care provision (Fig. 4).

\section{Discussion}

This paper described the processes involved in establishing the Korean CBPC model developed in a pilot project implemented in Busan Metropolitan City. This model was developed by surveying the regional characteristics and community demand for palliative care to identify the business goals and service content, establishing an operational infrastructure, and establishing a service delivery system.

During the six years of the pilot project before the CBPC project was implemented, the service provision teams' capacity was developed, the scope and level of service was determined, the patient referral and consortium systems were established in various institutions, and volunteer training and community education were provided to induce community participation. Consequently, the city health administrators recognized that the pilot project, which was based on public health centers in Busan that had an insufficient number of palliative care beds, increased the accessibility of end-of-life care for home-based cancer patients, including those belonging to the highly vulnerable low-income class, and ensured effective utilization of healthcare resources. In 2015, a CBPCC was established as the project was expanded to include public health centers across Busan. The model development team performed service management functions in the CBPCC while developing the model.

Public health centers served as regional hubs in the CBPC project, and each public health center established a palliative care team that functioned as a service provision team. Palliative nurses and social workers from the service provision teams were dispatched by the CBPCC, and other support was provided by public health center personnel by adjusting their job responsibilities. The advantage of this structure was that additional investment was not required to allow public health centers to implement palliative care projects. Moreover, service quality management was possible via the organic relationships between the service management and provision teams, which reduced the burden community member health placed on public health centers that implemented a range of other regional healthcare projects.

The project's service support team played an advisory role in providing administrative, financial, and technological consultation. The research support committee, based at the 
Health Improvement Department of Busan, regional cancer centers, and the Social Service Network, provided support and discussed the issues concerning project implementation. The medical support committee was responsible for educating physicians in public health centers to ensure that the palliative care recipients received drug treatment and medical support when required and provide consultation regarding drug treatment. The working committee consisted of the officers managing the project at public health centers, and it contributed to service standardization and improvements in task effectiveness across all public health centers via regulation and discussion regarding the issues concerning everyday palliative care tasks. The most difficult aspect of implementing the CBPC project was persuading public health centers to agree to implement a palliative care project and provide education regarding the provision of general information and an awareness of end-of-life care to physicians in public health centers and the officers managing the projects. In executing the project, the working and medical support committees played important roles in addressing the challenges, implementing the project effectively, and increasing service satisfaction.

The main pathway for palliative care recipient referral was accessed via project teams for home-based cancer patients and healthcare home-visit teams in public health centers. The educational unit of the service management team educated periodically healthcare home-visit teams and nurses working on projects for home-based cancer patients at public health centers to ensure they would refer potential palliative care recipients to a palliative nurse. Service provision teams provided home-visit services for the referred patients and co-managed these services with healthcare home-visit teams when necessary to guarantee quality services for recipients and effectively utilize the resources available at public health centers. Before the project was implemented, patients receiving end-of-life care and were registered at public health centers received intermittent healthcare rather than palliative care from nurses in healthcare home-visit teams [11]. Specifically, home-based cancer patients registered at public health centers and elderly patients with chronic illnesses registered with healthcare home-visit teams belonged mainly to a highly vulnerable, low-income class and did not receive quality medical services [12]. Therefore, the CBPC project could be evaluated positively as a new health management project implemented by public health centers that provides quality palliative care for low-income community members.

A consortium system was established to ensure that palliative care recipients requiring inpatient medical service were admitted to a consortium palliative medical center and were referred back to service provision teams after discharge to receive continuous palliative care. In 2015, there were only 87 palliative beds in Busan Metropolitan City; therefore, the capacity for inpatient medical care was insufficient [9]. Since July 2015, when the healthcare payment system began to cover inpatient palliative care, the waiting period for admission increased, and prompt admission for the inpatient treatment of case management recipients is difficult when their symptoms deteriorate. Accordingly, the provision of a medical support system, in which physicians in public health centers prescribe drug therapy to manage palliative care of the recipients' symptoms, is considered essential to help patients live at home for longer. On the other hand, this is currently impossible in Korea, where public institutions, such as public health centers, issue prescriptions for palliative care recipients. Therefore, the model development team will develop an assessment system and guidelines regarding symptoms and drug use in palliative care for physicians in public health centers.

To increase the quality of palliative care recipient case management, palliative nurses dispatched to service provision teams in public health centers held weekly case management meetings with the service management team. The objective of the CBPC project was to develop a practical model in which services are provided based on the public health centers' circumstances and palliative care principles. Accordingly, the CBPCC dispatched palliative nurses and social workers trained in the principles of palliative care to ensure that the palliative care project would not be burdensome to public health centers that had already implemented a range of other regional healthcare projects. Palliative nurses and social workers dispatched from the CBPCC were the project's primary service providers, and the service management team's education unit was responsible for the development and provision of educational programs designed to increase capacity.

The CBPC model is significant in that it suggests a new palliative care system for the consideration of policies regarding end-of-life treatment in Korea, where the health insurance program currently covers inpatient palliative care for end-stage cancer patients. In particular, the CBPC project helped expand the pool of professionals specializing in palliative care by supporting the standardization of palliative care education for service provision teams in public health centers. The system is based on the WHO's concept of primary palliative care, in which palliative care provided by public medical centers should be cost-effective and accessible to the entire community [13]. 


\section{Conclusion}

The CBPC model is expected to ultimately contribute to improvements in the National Quality of Death Index as a new means of providing universal end-of-life care in an aging society [14]. Therefore, the potential of the CBPC model presented herein and implemented in Korea should be evaluated by research and the development of outcome measures.

\section{Conflicts of Interest}

Conflict of interest relevant to this article was not reported.

\section{Acknowledgments}

This research was supported by a grant from the Korea Health Technology R\&D Project through the Korea Health Industry Development Institute (KHIDI), funded by the Ministry of Health \& Welfare, Republic of Korea (grant number: HI14C3399).

\section{References}

1. Ministry of Health and Welfare. Terminal cancer patient hospice utilization [Internet]. Sejong: Ministry of Health and Welfare; 2015 [cited 2016 Jan 14]. Available from: http://www. mohw.go.kr/front_new/sch/index.jsp.

2. Jung KW, Won YJ, Kong HJ, Oh CM, Cho H, Lee DH, et al. Cancer statistics in Korea: incidence, mortality, survival, and prevalence in 2012. Cancer Res Treat. 2015;47:127-41.

3. Ministry of Health and Welfare. Establish a legal basis for hospice \& palliative care and withdraw life-prolonging treatment [Internet]. Sejong: Ministry of Health and Welfare; 2016 [cited 2016 Feb 10]. Available from: http://www.mohw.go.kr/ front new / al / sal0301vw.jsp?PAR_MENU_ID $=04 \& M E N U \_I D=040$ $3 \&$ page $=1 \& C O N T \_S E Q=329848 \& S E A R C H K E Y=T I T L E \& S E A$ RCHVALUE.

4. Clark D, Wright M. The international observatory on end of life care: a global view of palliative care development. J Pain Symptom Manage. 2007;33:542-6.

5. Gwyther L, Brennan F, Harding R. Advancing palliative care as a human right. J Pain Symptom Manage. 2009;38:767-74.

6. Stjernsward J. Palliative care: the public health strategy. J Public Health Policy. 2007;28:42-55.

7. Institute for Research \& Industry Cooperation, Catholic University of Pusan. Report of hospice and palliative care project. Busan: Publication Seri Yun; 2014.

8. Statistics Korea. Annual report on the cause of death statistics 2014 [Internet]. Daejeon: Statistics Korea; 2015 [cited 2015 Dec
1]. Available from: http://kosis.kr/upsHtml/online/downSrvcFile.do?PUBCODE=YD\&FILE_NAME $=/$ YD / 06.xlsx\&SE $\mathrm{Q}=104$.

9. NHS England. Population-based needs assessment: specialist palliative care [Internet]. Leeds: NHS England; 2014 [cited 2016 Feb 10]. Available from: http://www.nescn.nhs.uk/ wp-content/ uploads / 2015/ 02 / Population-Needs-Assessment-Report-20143.pdf.

10. Kim SN, Choi SO, Kim YJ, Lee SR. Development of community health center-based hospice management model: pilot project at a community health center in Busan. Korean J Hosp Palliat Care. 2010;13:109-19.

11. Kang MH, Moon YS, Lee YJ, Kang YS, Kim HG, Lee GW, et al. Physical symptoms and psychiatric, social, spiritual and economical care needs of patients under home-based cancer service. Korean J Hosp Palliat Care. 2014;17:216-22.

12. Ministry of Health, Welfare and Family Affairs. The management for home-based cancer patients. Seoul: Ministry of Health, Welfare and Family Affairs; 2008.

13. Stjernsward J, Foley KM, Ferris FD. The public health strategy for palliative care. J Pain Symptom Manage. 2007;33:486-93.

14. The Economist Intelligence Unit. The 2015 Quality of Death Index: ranking palliative care across the world [Internet]. Economic Insights; 2015 [cited 2016 Jan 4]. Available from: http:// www.economistinsights.com/healthcare/analysis/ quality-death-index-2015. 